\title{
REFINO DE SILÍCIO POR TRATAMENTO A VÁCUO
}

\author{
André Alexandrino Lotto ' \\ João Batista Ferreira Neto ${ }^{2}$ \\ Marcelo Breda Mourão ${ }^{3}$
}

\section{Resumo}

Este trabalho visa investigar a remoção a vácuo de fósforo do silício grau metalúrgico (SiGM) $(98,5 \%$ a $99 \%$ de Si). Foram realizados experimentos de fusão em forno de indução a vácuo, variando parâmetros como temperatura, tempo e relação área exposta ao vácuo / volume de silício. Os resultados de análise química foram obtidos por plasma acoplado indutivamente (ICP) e avaliados com base em aspectos termodinâmicos e cinéticos da reação de vaporização do fósforo no silício. Obteve-se refino de 33 para cerca de I,5 ppm de P em três horas, concluindo-se que a etapa de evaporação é a etapa controladora do processo para os parâmetros de temperatura, pressão e agitação utilizados, e que o refino por este processo é tecnicamente viável.

Palavras-chave: Purificação; Silício; Vácuo; Fósforo.

\section{SILICON REFINING BY VACUUM TREATMENT}

\begin{abstract}
This work aims to investigate the phosphorus removal by vacuum from metallurgical grade silicon (MGSi) (98.5\% to $99 \% \mathrm{Si}$ ). Melting experiments were carried out in a vacuum induction furnace, varying parameters such as temperature, time and relation area exposed to the vacuum / volume of molten silicon. The results of chemical analysis were obtained by inductively coupled plasma (ICP), and evaluated based on thermodynamic and kinetic aspects of the reaction of vaporization of the phosphorus in the silicon. The phosphorus was decreased from 33 to approximately $1.5 \mathrm{ppm}$ after three hours of vacuum treatment, concluding that the evaporation step is the controlling step of the process for parameters of temperature, pressure and agitation used and refining by this process is technically feasible.
\end{abstract}

Keywords: Purification; Silicon; Vacuum; Phosphorus.

\section{INTRODUÇÃO}

A demanda mundial por energia elétrica vem crescendo vertiginosamente nas últimas décadas. Estima-se que até 2030 esta demanda aumente cerca de $55 \%$. Prevê-se um crescimento de $2,5 \%$ para $8,3 \%$ da geração de energia pelas novas fontes renováveis (solar, eólica, marés, e bioenergia). No geral, a eficiência das células fotovoltaicas é baixa, e seu preço elevado [I], mas este quadro está mudando com o crescimento da indústria e incentivos à produção que significam o ganho de economia de escala, para em um futuro próximo se atingir paridade entre o $\mathrm{kWh}$ gerado por fontes convencionais e o $\mathrm{kWh}$ gerado pela energia solar fotovoltaica.

O silício utilizado nas células fotovoltaicas, até a década de 1990, era fabricado com sobras prove- nientes da produção do silício de grau eletrônico, SiGE $(99,9999999 \% \mathrm{Si})$, que é muito mais puro que o exigido para a conversão de energia solar. Hoje existem processos específicos de produção de silício de grau solar (SiGS), seja por adaptação da rota de produção do SiGE, seja por rotas alternativas, como a de purificação pirometalúrgica do silício de grau metalúrgico, SiGM (98\% a 99,5\% de pureza). Este trabalho tem como objetivo explorar a técnica de fusão a vácuo do SiGM, visando a remoção de fósforo, que é difícil de se remover por solidificação direcional. A remoção de fósforo por fusão a vácuo poderia viabilizar a produção de SiGS para células fotovoltaicas de menor custo. Isto abriria uma frente de exportação para o Brasil, que é um dos maiores produtores de SiGM, com cerca de 200 mil

'Mestre em Engenharia Metalúrgica, Pesquisador, Centro de Tecnologia em Metalurgia e Materiais, Instituto de Pesquisas Tecnológicas do Estado de São Paulo - IPT, São Paulo, SP, Brasil. E-mail: andrelotto@ipt.br

${ }^{2}$ Doutor em Engenharia Metalúrgica, Pesquisador, Centro de Tecnologia em Metalurgia e Materiais, Instituto de Pesquisas Tecnológicas do Estado de São Paulo - IPT, São Paulo, SP, Brasil. E-mail: jbfn@ipt.br

${ }^{3}$ Doutor em Engenharia Metalúrgica, Professor associado, Departamento de Engenharia Metalurgia e de Materiais, Escola Politécnica, Universidade de São Paulo - USP, São Paulo, SP, Brasil. E-mail: mbmourao@usp.br 
toneladas por ano, agregando valor ao produto. O SiGM é vendido a aproximadamente US $\$ 2,50 / \mathrm{kg}$, enquanto o SiGS varia entre US $\$ 30 / \mathrm{kg}$ e US $\$ 60 / \mathrm{kg}$, dependendo da situação do mercado e de sua qualidade.

O princípio do refino a vácuo consiste em favorecer a reação de evaporação dos elementos mais voláteis, deslocando o equilíbrio químico no sentido da formação das fases gasosas [2]. A Figura I apresenta as curvas de pressão de vapor de equilíbrio de alguns elementos puros em função da temperatura. Pode-se notar que o Si é menos volátil que a maioria dos elementos apresentados, principalmente em relação ao fósforo [3].

Os cálculos cinéticos de remoção de fósforo são dificultados pelo fato de o fósforo apresentar três espécies gasosas principais: $\mathrm{P}_{1} \mathrm{P}_{2}$ e $\mathrm{P}_{4}$ [4]. Alguns pesquisadores estudaram as propriedades termodinâmicas do sistema Si-P. Miki et al. [5] chegaram a variação de energia livre de Gibbs apresentada na Equação I:

$$
P_{(\% \text { massa no si) }}=1 / 2 P_{2(g)} \quad \Delta G^{0}=139000-43,4 T[\mathrm{~J} / \mathrm{mol}](\mathrm{I})
$$

Zaitsev et al. [6] por um método e faixa de composição química diferente chegaram a variação de energia livre de Gibbs apresentada na Equação 2:

$$
P_{(\% \text { massa no Si) }}=1 / 2 P_{2(g)} \quad \Delta G^{0}=99500-29,46 \mathrm{~T} \quad[\mathrm{~J} / \mathrm{mol}](2)
$$

Com estes valores de energia livre de Gibbs e através da constante de equilíbrio é possível deduzir que a I.873K $\left(1.600^{\circ} \mathrm{C}\right)$ a espécie dominante na fase gasosa para concentrações de $\mathrm{P}$ menores que 63 ppm é a monoatômica
(P) para os dados de Miki et al. [5] e abaixo de 26 ppm para os dados de Zaitsev et al. [6].

Muitos pesquisadores vêm estudando a remoção de fósforo do silício sob vácuo. Suzuki et al. [7] reduziram o teor de fósforo de SiGM de 32 para 6 a 7 ppm em 21.600 s (6 horas) de tratamento a $1.823 \mathrm{~K}\left(1.550^{\circ} \mathrm{C}\right)$. Foi relatada também remoção de $\mathrm{Ca}$ e Al. Concluíram que a remoção de $\mathrm{Pe}$ Ca são controladas pela difusão destes elementos do líquido até a interface líquido/gás. Yuge et al. [8] trataram silício líquido sob vácuo de $8,0 \cdot 10^{-3}$ a $3,6 \cdot 10^{-2} \mathrm{~Pa}$ entre $1.722 \mathrm{~K}$ a $1.915 \mathrm{~K}\left(1.449^{\circ} \mathrm{C}\right.$ a $\left.1.642^{\circ} \mathrm{C}\right)$, e sob vácuo de $4 \mathrm{a}$ $190 \mathrm{~Pa}$ entre $1.733 \mathrm{~K}$ a $1.823 \mathrm{~K}\left(1.460^{\circ} \mathrm{C}\right.$ a $\left.1.550^{\circ} \mathrm{C}\right)$, obtendo a seguinte expressão para a variação do coeficiente de transferência de massa de fósforo $\left(k_{\mathrm{p}}\right)$ com a temperatura: In $k_{p}=-2,29-15.600 T^{-1}(m / s)$. Zheng et al. [9] obtiveram uma redução de $460 \mathrm{ppm}$ para $10 \mathrm{ppm}$ sob vácuo de $0,6 \mathrm{~Pa}$ a $0,8 \mathrm{~Pa}$ e $1.873 \mathrm{~K}\left(1.600^{\circ} \mathrm{C}\right)$ durante $3.600 \mathrm{~s}$ (I hora) e de I 5 ppm para $0,08 \mathrm{ppm}$ sob vácuo de $0,035 \mathrm{~Pa}$ a $0, \mathrm{I} \mathrm{Pa}$ e temperatura entre $1.773 \mathrm{~K}$ e $1.873 \mathrm{~K}\left(1.500^{\circ} \mathrm{C}\right.$ e $\left.1.600^{\circ} \mathrm{C}\right)$ em $7.200 \mathrm{~s}$ ( 2 horas) de tratamento. Safarian e Tangstad [ I0] conseguiram refino de $16,66 \mathrm{ppm}$ para $1,5 \mathrm{ppm}$ em $18.000 \mathrm{~s}$ (5 horas). Encontraram $k_{p}$ de $2,28.10^{-6} \mathrm{~m} / \mathrm{s}$ a $1.773 \mathrm{~K}$ $\left(1.500^{\circ} \mathrm{C}\right)$ e $4,93.10^{-6} \mathrm{~m} / \mathrm{s}$ a $1.873 \mathrm{~K}\left(1.600^{\circ} \mathrm{C}\right)$ e concluíram que a transferência de massa do fósforo em banhos agitados pela indução é grande, não constituindo um limitante para a cinética de remoção de fósforo, e que o refino é controlado tanto pela reação de evaporação na superfície quanto pelo transporte na fase gasosa, com esta última se tornando mais importante com pressões e temperaturas mais altas.

A evaporação preferencial de soluto deve ser ainda analisada sob um aspecto cinético. A razão das velocidades

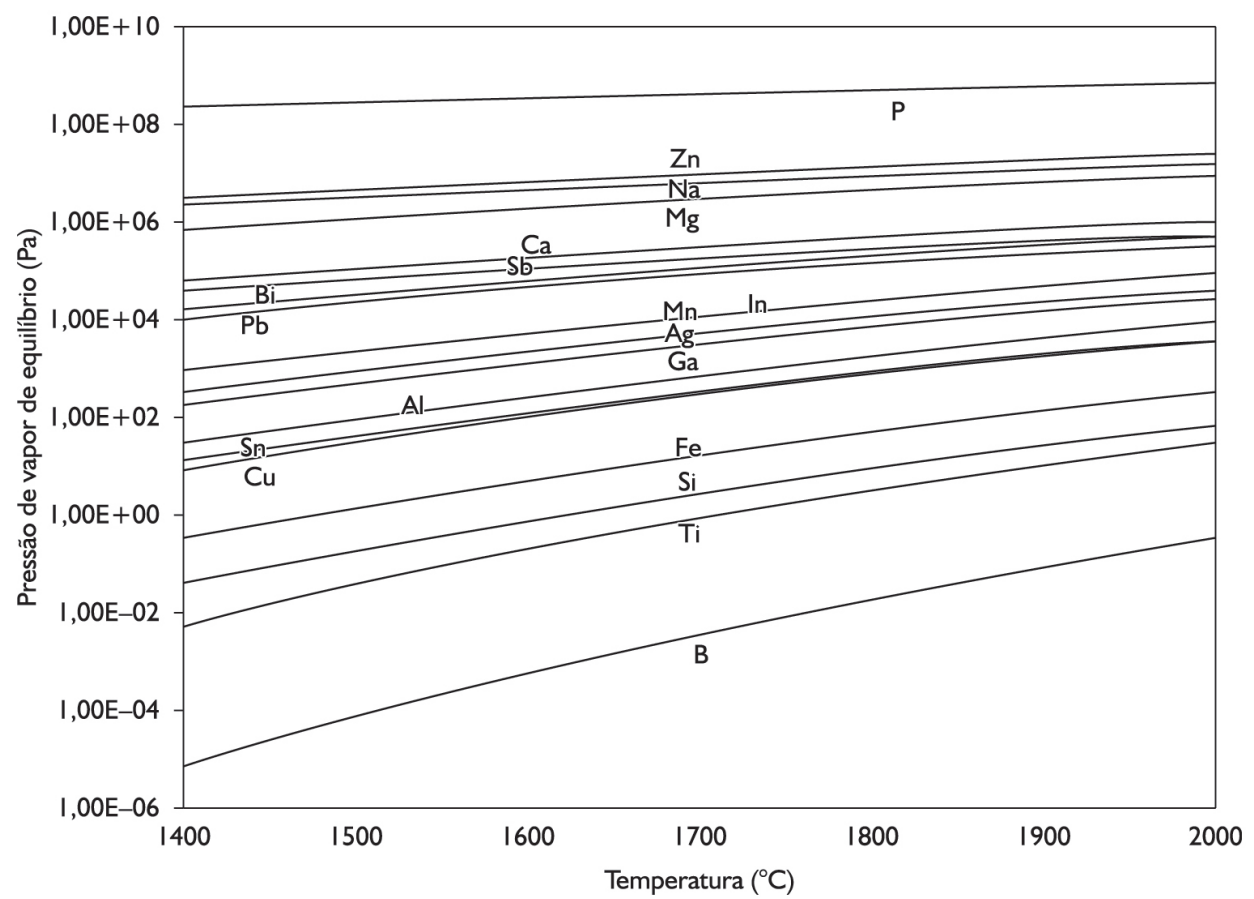

Figura I. Curvas de variação da pressão de vapor padrão de substâncias puras em função da temperatura. Adaptado de Safarian e Tangstad [3]. 
de remoção de soluto e solvente deve exceder a razão das respectivas concentrações no banho. Neste trabalho serão consideradas as seguintes etapas básicas de transporte em líquidos aquecidos por indução:

- Transporte de soluto na fase líquida até próximo da superfície do líquido;

- Transporte através de uma camada limite estagnante na superfície do líquido;

- Reação de evaporação na interface metal/gás;

- Transporte do soluto na fase gasosa até uma superfície de condensação; e

- Condensação ou remoção das espécies gasosas pelas bombas de vácuo.

Deve ainda ser analisada a evaporação de silício, já que a concentração de soluto é muito baixa, e a etapa de transporte do soluto na fase gasosa pode concorrer com o vapor de solvente. As relações cinéticas podem ser expressas pela Equação 3 [I I]:

$$
-\frac{d C}{d t}=k_{i} \cdot \frac{A}{V} \cdot\left(C-C_{e}\right)^{n}
$$

Onde $k_{i}$ é o coeficiente de transporte global da reação para a espécie i $\left(\mathrm{Si}, \mathrm{P}\right.$ ou $\mathrm{P}_{2}$ ), C é a concentração de fósforo no tempo $t, C_{e}$ é a concentração de equilíbrio do fósforo, $A$ é a área de superfície de evaporação, $V$ é o volume de líquido e $n$ é a ordem da reação. A literatura indica que a evaporação de $P$ segue a reação de primeira ordem e a evaporação de $\mathrm{P}_{2}$ segue a de segunda ordem. $\mathrm{O}$ coeficiente global de transporte $k_{p}$ pode ser definido pela Equação 4:

$$
\frac{\mathrm{l}}{\mathrm{k}_{\mathrm{P}}}=\frac{\mathrm{l}}{\mathrm{k}_{\mathrm{L}}}+\frac{\mathrm{l}}{\mathrm{k}_{\mathrm{E}}}+\frac{\mathrm{l}}{\mathrm{k}_{\mathrm{G}}} \quad[\mathrm{s} / \mathrm{m}]
$$

Onde $k_{L}, k_{E}$ e $k_{G}$ são as constantes de transporte na fase líquida, na evaporação e na fase gasosa, respectivamente. Para determinar qual a etapa controladora do processo, esses coeficientes devem ser analisados separadamente. $k_{p}$ pode ser determinado teoricamente pela equação acima, ou experimentalmente, assumindo-se que a reação seja de primeira ordem. Neste trabalho considerou-se que as reações são de primeira ordem devido à baixa concentração inicial de fósforo.

Safarian e Tangstad [3] desenvolveram um modelamento matemático para prever a cinética de remoção de impurezas de silício fundido a vácuo.

Neste trabalho foi utilizado um modelo desenvolvido por Zheng et al. [4] para prever o comportamento das impurezas durante o refino. $O$ modelo foi adaptado para os parâmetros utilizados nos experimentos e algumas estimativas baseadas na literatura foram feitas. O modelo se divide basicamente na análise de evaporação de silício, que é o elemento principal na fase gasosa devido à baixíssima concentração das impurezas no líquido, além do transporte da espécie $\mathrm{P}$ nas fases líquida e gasosa e sua evaporação.

\section{I.I Evaporação de Si}

O silício pode ser tratado como substância pura, e pode-se considerar que a evaporação deste elemento se dá em toda a superfície do banho. Deve-se levar em conta também a etapa de transporte na fase gasosa. As considerações referentes a estas etapas podem ser encontradas no trabalho de Zheng et al. [4] Pode-se considerar que a evaporação de silício ocorre a uma taxa constante quando não é considerado o efeito das impurezas. Assim, a evaporação de silício em função do tempo $\left(\mathrm{J}_{\mathrm{S}_{\mathrm{i}}}\right)$ é descrita pela Equação 5:

$$
\mathrm{J}_{\mathrm{Si}}=\mathrm{j}_{\mathrm{Si}} \cdot\left(\mathrm{t}-\mathrm{t}_{0}\right) \cdot M_{\mathrm{Si}}=\mathrm{A} \cdot \mathrm{M}_{\mathrm{Si}} \cdot \frac{\mathrm{k}_{\mathrm{Si}}}{\mathrm{RT}} \cdot \mathrm{p}_{\mathrm{Si}}^{\mathrm{e}} \cdot\left(\mathrm{t}-\mathrm{t}_{0}\right) \quad[\mathrm{kg}]
$$

Onde $\mathrm{j}_{\mathrm{Si}}$ é o fluxo de $\mathrm{Si}$ em equilíbrio, $\mathrm{k}_{\mathrm{Si}}$ é o coeficiente de transferência de massa do $\mathrm{Si}$, $\mathrm{p}_{\mathrm{Si}}^{\mathrm{e}}$ é a pressão parcial de equílbrio do $\mathrm{Si}, \mathrm{A}$ é a área superficial do banho e $\mathrm{M}_{\mathrm{Si}}$ é a massa molecular do $\mathrm{Si}$.

\section{I.2 Evaporação da Espécie Gasosa P}

Como o banho sofre forte agitação devido a indução, assume-se que a etapa de transporte de soluto na fase líquida até próximo da superfície do líquido não é limitante para a evaporação da espécie gasosa P. Assume-se que a etapa de condensação também não é limitante porque a condensação ocorre numa grande área de superfície fria e os elementos evaporados são rapidamente retirados do sistema pelas bombas de vácuo [4].

\section{I.2. I Etapa 2: transporte através da camada limite estagnante na superfície do líquido $\left(k_{L}\right)$}

Muitas teorias foram propostas para modelar o coeficiente de transporte na fase líquida até a interface líquido/gás $\left(\mathrm{k}_{\mathrm{L}}\right)$. A mais simples defende que a agitação convectiva causada pelo campo magnético mantém uma concentração homogênea das espécies envolvidas no banho exceto por uma camada limite junto à interface líquido/gás, onde ocorre o gradiente de composição [I I ]. Neste caso, pode-se empregar, por exemplo, o modelo de Machlin, o qual é indicado para velocidades altas como as geradas por fornos de indução. O coeficiente de transporte pode assim ser expresso pela Equação 6 [4].

$$
\mathrm{k}_{\mathrm{L}}=\sqrt{\left(\frac{8 \cdot \mathrm{D}_{\mathrm{P}(\mathrm{l})} \cdot \mathrm{v}_{\mathrm{m}}}{\pi \cdot \mathrm{r}}\right)}[\mathrm{m} / \mathrm{s}]
$$

Onde $D_{\mathrm{P}()}$ é o coeficiente de difusão do fósforo no líquido, $v_{\mathrm{m}}$ é a velocidade de convecção do líquido e $r$ é o raio da superfície circular do banho.

Para obtenção deste coeficiente, a dificuldade do cálculo está na avaliação da velocidade de convecção do líquido, que é criada pela interação entre as correntes 
induzidas e o campo magnético. Existem modelos para - cálculo dessa velocidade baseado nas características do campo magnético e do metal, porém a dificuldade em se medir esse campo, principalmente com a interação com o cadinho, impossibilita análises mais profundas desse parâmetro. Alguns autores utilizam a velocidade de $0,1 \mathrm{~m} / \mathrm{s}$ estimada por Machlin e prevista teoricamente por Tarapore e Evans [4].

\subsubsection{Etapa 3: reação de evaporação na interface metal/gás (kE)}

A evaporação de $P$ na interface pode ser tratada utilizando-se a equação de Hertz-Langmuir-Knudsen e obtendo-se a seguinte constante representada pela Equação 7:

$$
k_{E}=\frac{100 \cdot \alpha \cdot p_{P}^{*} \cdot f_{P}}{\rho_{S i}} \cdot \sqrt{\frac{M_{P}}{2 \pi R T}}[\mathrm{~m} / \mathrm{s}]
$$

Onde $\mathrm{P}_{\mathrm{P}}^{*}$ é a pressão de vapor de $\mathrm{P}$ no silício líquido em diluição infinita [4].

\section{I.2.3 Etapa 4: transporte do soluto na fase gasosa até uma superfície de condensação (kG)}

O fluxo total de $P$ na fase gasosa é representado pela Equação 8, e é igual a somatória do fluxo difusivo e do fluxo convectivo de $\mathrm{P}$. Os fluxos das espécies $\mathrm{P}$ e $\mathrm{P}_{2}$ podem ser ignorados se comparados ao fluxo de $\mathrm{Si}$.

$$
\mathrm{k}_{\mathrm{G}}=\frac{\mathrm{D}_{\mathrm{P}(\mathrm{g})}}{\delta} \cdot \frac{\xi_{\mathrm{P}}}{\mathrm{e}^{\xi_{\mathrm{p}}-1}} \quad[\mathrm{~m} / \mathrm{s}]
$$

Onde $x_{p}$ é o fator de efeito de difusão do vapor de silício na espécie $P, D_{P(g)}$ é o coeficiente de difusão do fósforo no líquido e $\delta$ é a distância entre a superfície do banho até a superfície de condensação [4]. Nesta etapa entra o equacionamento da evaporação de Si.

\section{METODOLOGIA}

Foi utilizado um forno tipo Bridgman, de fusão por indução a vácuo de 30 kVA e $7 \mathrm{kHz}$ do Centro de Tecnologia em Metalurgia e Materiais (CTMM) do IPT. O forno possui uma bomba mecânica de palhetas rotativas, uma bomba rotativa tipo "roots" e uma bomba difusora, que em conjunto, permitem atingir pressão de até 0,02 Pa. A medição da pressão é feita por meio de sensores tipo "pirani" e catodo frio. A medição de temperatura foi feita por pirômetro óptico. As amostragens durante os ensaios foram realizadas com amostradores de grafite. Nos experimentos realizados as seguintes variáveis foram definidas:

- temperatura de tratamento do banho de $1.973 \mathrm{~K} \mathrm{e}$ $2.023 \mathrm{~K}\left(1.650^{\circ} \mathrm{C}\right.$ e $\left.1.750^{\circ} \mathrm{C}\right)$;

- tempo de tratamento de até $10.800 \mathrm{~s}$ (até $3 \mathrm{~h}$ );

- duas relações área/volume do banho de Si de Xe 3X;

- nível de vácuo da câmara entre 0,08 a $2 \mathrm{~Pa}$.

Foram retiradas três amostras com o tempo por ensaio. Foi utilizado como matéria-prima SiGM fornecido pela Companhia Ferroligas Minas Gerais (Minasligas), com teor de fósforo inicial de $33 \pm 6 \mathrm{ppm}$. A Tabela I apresenta a composição química do lote utilizado nos ensaios.

Os cadinhos utilizados foram cadinhos de grafita de alta densidade $\left( \pm 1.800 \mathrm{~kg} / \mathrm{m}^{3}\right)$. As amostras foram analisadas por espectrometria de emissão atômica por plasma acoplado indutivamente, ou ICP-OES no laboratório de análises químicas do CTMM.

\section{RESULTADOS}

A Tabela 2 apresenta os principais resultados obtidos nos ensaios para avaliar o efeito da temperatura, do tempo de tratamento, e da área de superfície do banho (relação A/N).

A Tabela 3 apresenta os resultados de análise química para outros elementos normalmente encontrados no silício grau metalúrgico, tais como: alumínio, cálcio, magnésio, manganês e zinco.

\section{DISCUSSÃO}

Como pode ser visto nas Tabelas 2 e 3, houve significativa redução nos teores de alguns elementos com o tempo. Os resultados tendem a ser melhores para maiores relações $\mathrm{A} / \mathrm{V}$. Os resultados não mostram grande variação com as diferentes temperaturas e pressões utilizadas.

Os resultados experimentais e as curvas do modelamento de Zheng et al. [4] para os parâmetros utilizados nos ensaios são comparados a seguir nas Figuras 2 a 5 . O mesmo modelo foi plotado utilizando os dados termodinâmicos de Miki et al. [5] e os de Zaitsev et al. [6] considerando somente evaporação de $P$.

Pode-se observar que o modelo baseado nos dados termodinâmicos de Miki et al. [5] descreve melhor os resultados experimentais obtidos. A Tabela 4 mostra os coeficientes de transporte de massa para cada etapa

Tabela I. Composição do silício utilizado (em ppm)

\begin{tabular}{cccccccccc}
\hline $\mathrm{B}$ & $10 \pm \mathrm{I}$ & $\mathrm{Cr}$ & $69 \pm 24$ & $\mathrm{Zr}$ & $6 \pm 2$ & $\mathrm{P}$ & $33 \pm 6$ & $\mathrm{Na}$ & $<3$ \\
$\mathrm{Mn}$ & $45 \pm 8$ & $\mathrm{Ni}$ & $39 \pm \mathrm{I}$ & $\mathrm{Zn}$ & $<\mathrm{I}$ & $\mathrm{Fe}$ & $2.800 \pm 30$ & $\mathrm{Cu}$ & $106 \pm 42$ \\
$\mathrm{Ca}$ & $120 \pm 4$ & $\mathrm{Ti}$ & $75 \pm 25$ & $\mathrm{~V}$ & $4 \pm \mathrm{I}$ & $\mathrm{Mg}$ & $4 \pm \mathrm{I}$ & $\mathrm{Al}$ & $234 \pm 96$ \\
\hline
\end{tabular}


Tabela 2. Teor de $\mathrm{P}$ (em ppm) da matéria prima e das amostras coletadas durante os ensaios conduzidos em forno de indução sob vácuo em função da temperatura e da relação área/volume

\begin{tabular}{|c|c|c|c|c|c|}
\hline \multirow[b]{2}{*}{ Pressão média } & \multicolumn{3}{|c|}{$1.973 \mathrm{~K}\left(1.650^{\circ} \mathrm{C}\right)$} & \multicolumn{2}{|c|}{$2.023 \mathrm{~K}\left(1.750^{\circ} \mathrm{C}\right)$} \\
\hline & $0,28 \mathrm{~Pa}$ & $0,08 \mathrm{~Pa}$ & $0,27 \mathrm{~Pa}$ & $0,3 \mathrm{~Pa}$ & 2,05 Pa \\
\hline Tempo & $3 x$ & $\mathbf{x}$ & $3 x$ & $3 x$ & $\mathbf{x}$ \\
\hline $0 \mathrm{~s}$ (P inicial) & $33 \pm 6$ & $33 \pm 6$ & $33 \pm 6$ & $33 \pm 6$ & $33 \pm 6$ \\
\hline $1.800 \mathrm{~s}$ & $18 \pm 4$ & $16 \pm 1$ & $9 \pm 3$ & $10 \pm 1$ & $\mathrm{II}, 2 \pm 0,4$ \\
\hline $5.400 \mathrm{~s}$ & $2,2 \pm 0,4$ & $16 \pm 2$ & $3,3 \pm 0,8$ & $2,1 \pm 0,6$ & $12,7 \pm 2,3$ \\
\hline $10.800 \mathrm{~s}$ & $\mathrm{I}, 5 \pm 0,2$ & $10,3 \pm 1,5$ & $2,0 \pm 0,3$ & $2,0 \pm 0,4$ & $5,1 \pm 0,3$ \\
\hline
\end{tabular}

Tabela 3. Variação da composição de outros elementos voláteis durante refino de fósforo (em ppm)

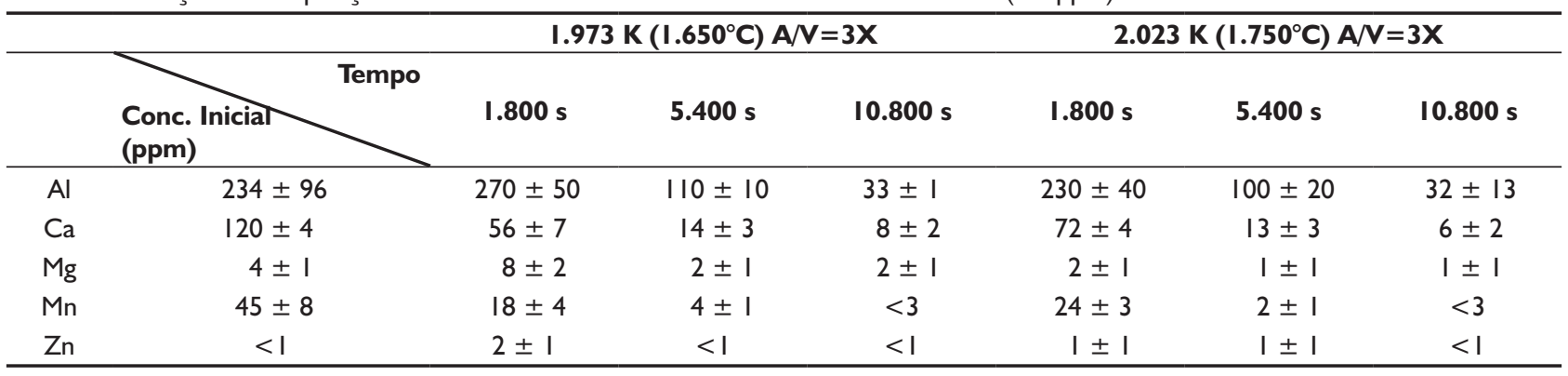

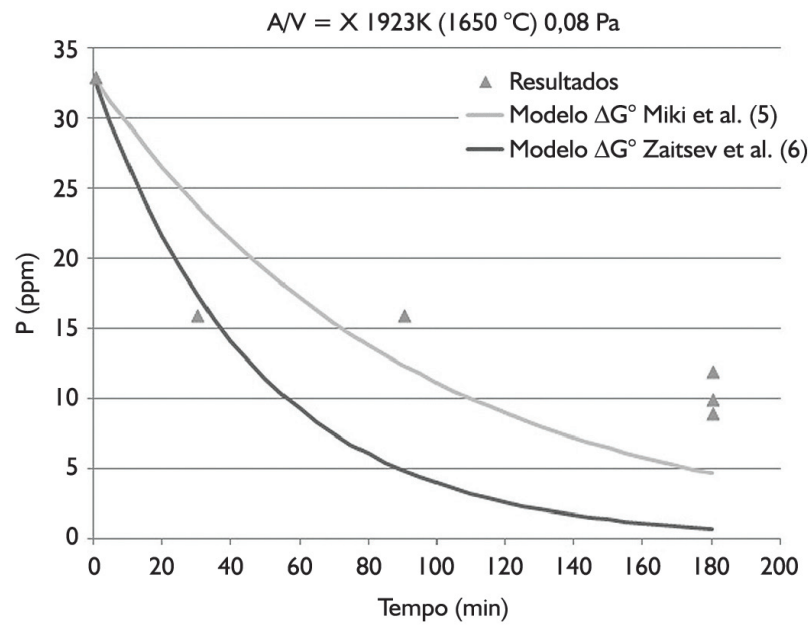

Figura 2. Resultados experimentais versus modelo para dois ensaios iguais.

e global, tanto obtido experimentalmente, quanto pelo modelo.

Pode-se observar que na maioria dos casos $\circ \mathrm{k}_{\mathrm{p}}$ experimental é menor que o teórico. Isso também pode ser observado nas curvas experimentais (Figuras 2 a 5), que são na maioria dos casos cineticamente mais lentas na remoção de $\mathrm{P}$ que as do modelo. Possivelmente, na etapa de transporte na camada estagnante do líquido a velocidade de convecção do líquido é menor que a estimada.

Pela Tabela 4, pode-se observar que a etapa de evaporação é a etapa controladora do processo, mas conforme o nível de vácuo da câmara e a temperatura

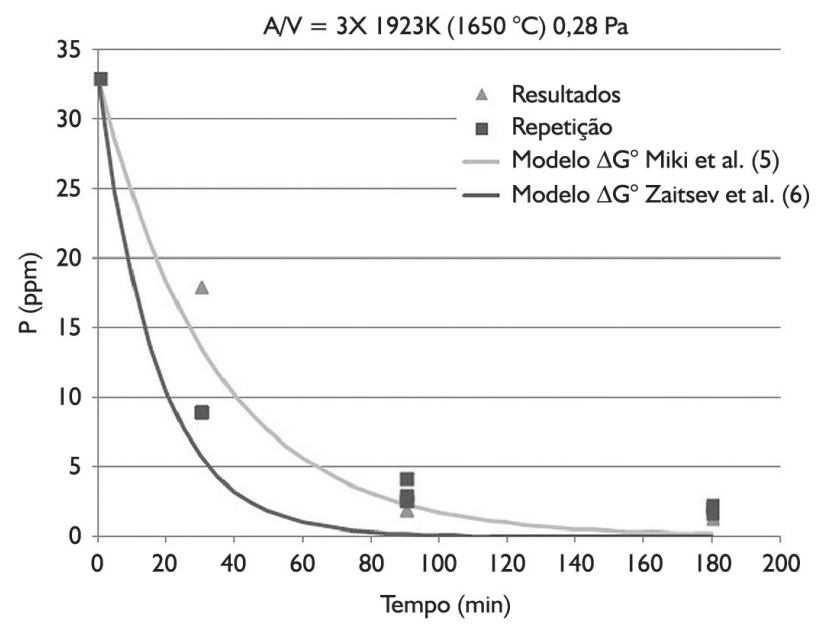

Figura 3. Resultados experimentais versus modelo.

sobem a etapa controladora passa a ser transporte no gás, concordando com o que foi observado por Safarian e Tangstad [10]. A Tabela 5 apresenta uma comparação entre coeficientes globais de transporte encontrados por alguns autores na literatura para diferentes parâmetros e os obtidos experimentalmente neste trabalho para as temperaturas de $1.923 \mathrm{Ke} 2.023 \mathrm{~K}\left(1.650^{\circ} \mathrm{C}\right.$ e $\left.1.750^{\circ} \mathrm{C}\right)$, pressão de $0,3 \mathrm{~Pa}$ e relação $\mathrm{A} / \mathrm{V}$ de $3 \mathrm{X}$. Pode-se observar que os coeficientes experimentais obtidos são mais próximos daqueles obtidos por Safarian e Tangstad [ 10 ] e as diferenças encontradas podem ser decorrentes de diferentes condi- 


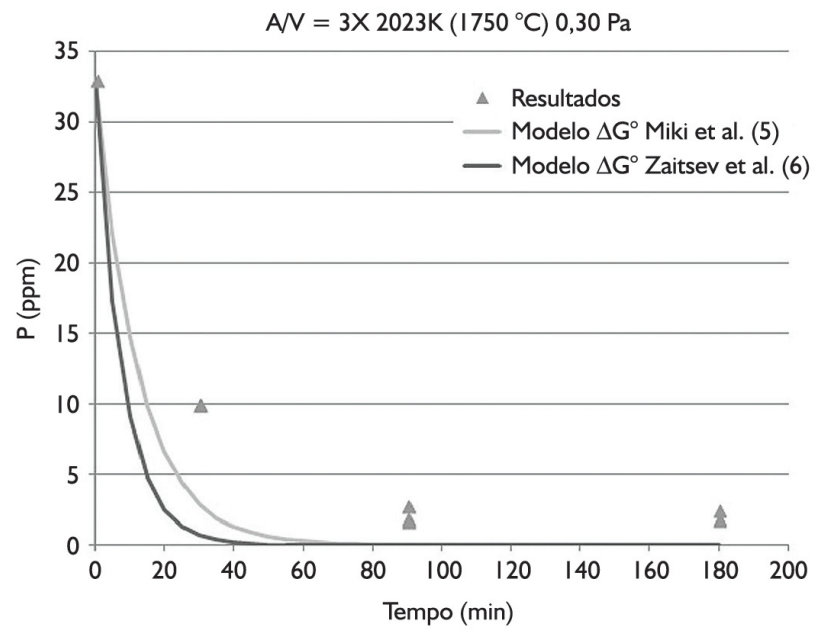

Figura 4. Resultados experimentais versus modelo.

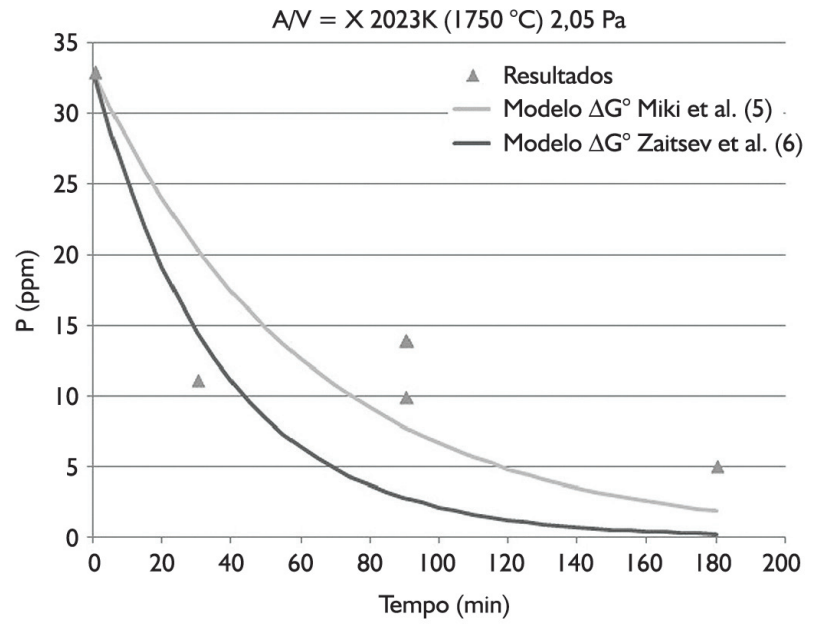

Figura 5. Resultados experimentais versus modelo.

Tabela 4. Comparação entre os coeficientes globais de transporte experimental e teórico $(\mathrm{em} \mathrm{m} / \mathrm{s})$

\begin{tabular}{cccccc}
\hline Experimento & $\mathbf{k}_{\mathrm{P}}$ experimental & $\mathbf{k}_{\mathrm{L}}$ & $\mathbf{k}_{\mathrm{E}}$ & $\mathbf{k}_{\mathrm{G}}$ & $\mathbf{k}_{\mathrm{P}}$ teórico \\
\hline $\mathrm{A} / \mathrm{V}=3 \times 1.923 \mathrm{~K} 0,28 \mathrm{~Pa}$ & $7,0 \cdot 10^{-6}\left(\mathrm{R}^{2}=0,8443\right)$ & $\mathrm{I}, 6 \cdot 10^{-4}$ & $\mathrm{I}, 6 \cdot 10^{-5}$ & $9,6 \cdot 10^{-5}$ & $\mathrm{I}, 3 \cdot 10^{-5}$ \\
$\mathrm{~A} / \mathrm{N}=\mathrm{X} 1.923 \mathrm{~K} 0,08 \mathrm{~Pa}$ & $7,0 \cdot 10^{-6}\left(\mathrm{R}^{2}=0,7676\right)$ & $1,6 \cdot 10^{-4}$ & $1,6 \cdot 10^{-5}$ & $3,4 \cdot 10^{-4}$ & $1,4 \cdot 10^{-5}$ \\
$\mathrm{~A} / \mathrm{N}=3 \times 2.023 \mathrm{~K} 0,30 \mathrm{~Pa}$ & $4,0 \cdot 10^{-6}\left(\mathrm{R}^{2}=0,86 \mathrm{I} 2\right)$ & $1,6 \cdot 10^{-4}$ & $5,4 \cdot 10^{-5}$ & $3,0 \cdot 10^{-4}$ & $3,6 \cdot 10^{-5}$ \\
$\mathrm{~A} / \mathrm{V}=\mathrm{X} 2.023 \mathrm{~K} 2,05 \mathrm{~Pa}$ & $7,0 \cdot 10^{-4}\left(\mathrm{R}^{2}=0,86 \mathrm{I}\right)$ & $1,6 \cdot 10^{-4}$ & $5,4 \cdot 10^{-5}$ & $4,4 \cdot 10^{-5}$ & $2,1 \cdot 10^{-5}$ \\
\hline
\end{tabular}

Tabela 5. Comparação entre as médias dos coeficientes globais de transporte experimental por temperatura e coeficientes da literatura (em $\mathrm{m} / \mathrm{s}$ )

\begin{tabular}{ccccc}
\hline $\mathbf{k}_{\mathbf{p}}$ experimental & $\mathbf{k}_{\mathrm{p}}$ modelo & $\mathbf{k}_{\mathrm{p}}$ Suzuki et al. [7] & $\mathbf{k}_{\mathrm{p}}$ Yuge et al. [8] & $\mathbf{k}_{\mathrm{p}}$ Safarian e Tangstad [I0] \\
\hline $7,0 \cdot 10^{-6}(1.923 \mathrm{~K})$ & $1,3 \cdot 10^{-5}(1.923 \mathrm{~K})$ & $\mathrm{I}, 6 \cdot 10^{-5}(\mathrm{I} .723 \mathrm{~K})$ & $1,2 \cdot 10^{-5}(\mathrm{I} .723 \mathrm{~K})$ & $2,28 \cdot 10^{-6}(\mathrm{I} .773 \mathrm{~K})$ \\
$4,0 \cdot 10^{-6}(2.023 \mathrm{~K})$ & $2,8 \cdot 10^{-5}(2.023 \mathrm{~K})$ & $2,0 \cdot 10^{-5}(1.823 \mathrm{~K})$ & $1,9 \cdot 10^{-5}(1.823 \mathrm{~K})$ & $4,9 \cdot 10^{-6}(\mathrm{I} .873 \mathrm{~K})$ \\
& & & $3,0 \cdot 10^{-5}(1.923 \mathrm{~K})$ & \\
\hline
\end{tabular}

ções experimentais, principalmente temperatura, grau de agitação do banho e pressão na câmara.

\section{CONCLUSÃO}

Conclui-se que o processo de remoção de fósforo via fusão a vácuo é tecnicamente viável e que a etapa de evaporação é a etapa controladora do processo para os parâmetros de temperatura, pressão e agitação utilizados.
Este processo é promissor no refino de silício para a fabricação de células para conversão de energia solar.

\section{Agradecimentos}

Agradecemos a Companhia Ferroligas Minas Gerais (MinasLigas) e ao Banco Nacional de Desenvolvimento Econômico e Social (BNDES) pela oportunidade de desenvolvimento deste trabalho.

\section{REFERÊNCIAS}

I Centro de Gestão e Estudos Estratégicos. Materiais avançados no Brasil 2010-2022. Brasília: Centro de Gestão e Estudos Estratégicos; 2010.

2 Winkler OC, Bakish RA. Vacuum metallurgy. Amsterdã: Elsevier Publishing Company; I97I.

3 Safarian J, Tangstad M. Vacuum refining of molten silicon. Metallurgical and Materials Transactions. B, Process Metallurgy and Materials Processing Science. 20I2;43(6): I427-I445. http://dx.doi.org/I0. I007/s I I663-0I2-9728-I.

4 Zheng SS, Engh TA, Tangstad M, Luo XT. Numerical simulation of phosphorus removal from silicon by induction vacuum refining. Metallurgical and Materials Transactions. A, Physical Metallurgy and Materials Science. 20I I;42(8):22 I4-2225. http://dx.doi.org/I0.1007/s I I66 I-0 I I-062I-3. 
5 Miki T, Morita K, Sano N. Thermodynamics of phosphorus in molten silicon. Metallurgical and Materials Transactions. B, Process Metallurgy and Materials Processing Science. 1996;27(6):937-94I. http://dx.doi. org/I0.1007/s I I663-996-0007-x.

6 Zaitsev AI, Litvina AD, Shelkova NE. Thermodynamic Properties of Si-P Melts. High Temperature. 2001;39(2):227232. http://dx.doi.org/10.1023/A:10175707/5796.

7 Suzuki K, Sakaguchi K, Nakagiri T, Sano N. Gaseous removal of phosphorus and boron from molten silicon. Journal of the Japan Institute of Metals and Materials. 1990;54:161-167.

8 Yuge N, Hanazawa K, Nishikawa K, Terashima H. Removal of phosphorus, aluminum and calcium by evaporation in molten silicon. Journal of the Japan Institute of Metals and Materials. 1997;61:1086-1093.

9 Zheng SS, Safarian J, Seok S, Kim S, Merete T, Luo XT. Elimination of phosphorus vaporizing from molten silicon at finite reduced pressure. Transactions of Nonferrous Metals Society of China. 20I I;2I(3):697-702. http://dx.doi. org/10.1016/S1003-6326(II)60768-I.

10 Safarian J, Tangstad M. Kinetics and mechanism of phosphorus removal from silicon in vacuum induction refining. High Temperature Material Processes. 2012;31:73-81.

II Beneduce Neto F. Contribuição ao estudo das interações metal/revestimento em forno à indução sob vácuo [dissertação de mestrado]. São Paulo: Escola Politécnica, Universidade de São Paulo; 199|.

Recebido em: 18 Nov. 2013

Aceito em: 7 Ago. 2014 